\title{
Padrões espirométricos de resposta ao uso do broncodilatador em pacientes acometidos por doenças respiratórias
}

\author{
Spirometric patterns of response to bronchodilator use in patients affected by respiratory \\ diseases
}

\author{
Patrones espirométricos de respuesta al uso de broncodilatadores en pacientes con \\ enfermedades respiratorias
}

Lucas Vieira $^{1 *}$, Fernanda Majolo¹, Paula Michele Lohmann¹, Liana Johann¹, Guilherme Liberato da Silva ${ }^{1}$.

\section{RESUMO}

Objetivo: Estudar a resposta ao broncodilatador em pacientes acometidos por doenças respiratórias como asma e bronquite descrevendo as características clínicas avaliadas, bem como avaliar os diferentes padrões espirométricos expressados em patologias respiratórias encaminhadas para a espirometria. Métodos: Foram selecionados os prontuários e as espirometrias dos pacientes que apresentavam quadro de asma, bronquite ou rinite entre os anos de 2016-2019. Os testes espirométricos consistiram em uma etapa pré-broncodilatador e pós-broncodilatador. Foram utilizados os seguintes dados espirométricos: 1) capacidade vital forçada expiratória $(F V C)$, volume expiratório forçado no primeiro segundo $\left(F E V_{1}\right), V_{E F} / F V C$ Pré e Pósbroncodilatador. Resultados: Dos 34 pacientes avaliados neste estudo, $23(70 \%)$ tinham diagnóstico de asma e $9(28 \%)$ de bronquite e $1(2 \%)$ de rinite. Os asmáticos que apresentaram quadro clínico obstrutivo foi de 11 $(47,82 \%)$ juntamente com pacientes com bronquite, que também apresentaram, na sua maioria, quadro clínico obstrutivo (55,55\%). No estudo, 22 (67\%) não tiveram resposta ao broncodilatador. Dentre os asmáticos, préFVC\% e pós-FVC\% apresentaram diferença significativa $(p<0,0001)$. Conclusão: Considerando os pacientes com asma e bronquite, a expressão de FVC pós uso de broncodilatador demonstrou diferença significativa entre as duas patologias, tendo maior variação dos valores de $\mathrm{VEF}_{1}$ nos pacientes asmáticos.

Palavras-chave: Espirometria, Pneumopatias, Ambulatório.

\begin{abstract}
Objective: To study the response to bronchodilator in patients affected by respiratory diseases such as asthma and bronchitis, describing the clinical characteristics evaluated, as well to evaluate the different spirometric patterns expressed in respiratory pathologies referred to spirometry. Methods: Medical records and spirometry of patients with asthma, bronchitis or rhinitis between the years 2016-2019 were selected. The spirometric tests consisted of a pre-bronchodilator and post-bronchodilator stage. The following spirometric data were used: 1) forced expiratory vital capacity (FVC), forced expiratory volume in the first second (FEV 1 ), FEV $_{1} /$ FVC Pre and Post-bronchodilator. Results: Of the 34 patients evaluated in this study, 23 (70\%) had a diagnosis of asthma and $9(28 \%)$ of bronchitis and $1(2 \%)$ of rhinitis. Asthmatics who presented with an obstructive clinical condition was $11(47.82 \%)$ together with patients with bronchitis, who also mostly presented an obstructive clinical condition (55.55\%). In the study, 22 (67\%) had no response to the bronchodilator. Among asthmatics, pre-FVC\% and post-FVC\% showed a significant difference $(p<0.0001)$. Conclusion: Considering patients with asthma and bronchitis, the expression of FVC after bronchodilator use showed a significant difference between the two pathologies, with a greater variation in $\mathrm{FEV}_{1}$ values in asthmatic patients.
\end{abstract}

Key words: Spirometry, Lung diseases, Ambulatory.

1 Universidade do Vale do Taquari (Univates), Lajeado - RS. *E-mail: lucas.vieira@universo.univates.br 


\section{RESUMEN}

Objetivo: Estudiar la respuesta al broncodilatador en pacientes afectados por enfermedades respiratorias como asma y bronquitis, describiendo las características clínicas evaluadas, así como evaluar los diferentes patrones espirométricos expresados en patologías respiratorias referidas a la espirometría. Métodos: Se seleccionaron historias clínicas y espirometría de pacientes con asma, bronquitis o rinitis entre los años 20162019. Las pruebas espirométricas consistieron en un estadio prebroncodilatador y posbroncodilatador. Se utilizaron los siguientes datos espirométricos: 1) capacidad vital espiratoria forzada (FVC), volumen espiratorio forzado en el primer segundo (FEV1), FEV 1 / FVC Pre y Postbroncodilatador. Resultados: De los 34 pacientes evaluados en este estudio, $23(70 \%)$ tenían diagnóstico de asma y $9(28 \%)$ de bronquitis y $1(2 \%)$ de rinitis. Los asmáticos que presentaban un cuadro clínico obstructivo tenían $11(47,82 \%)$ junto con los pacientes con bronquitis, quienes también presentaban en su mayoría una condición clínica obstructiva $(55,55 \%)$. En el estudio, 22 (67\%) no respondieron al broncodilatador. Entre los asmáticos, el\% pre-FVC y el\% post-FVC mostraron una diferencia significativa $(p<0,0001)$. Conclusión: Considerando los pacientes con asma y bronquitis, la expresión de FVC después del uso de broncodilatador mostró una diferencia significativa entre las dos patologías, con una mayor variación en los valores del FEV ${ }_{1}$ en los pacientes asmáticos.

Palabras clave: Espirometría, Enfermedades pulmonares, Ambulatorio.

\section{INTRODUÇÃO}

As doenças respiratórias crônicas (DRCs) são um grupamento de patologias que causam a morte precoce de adultos em todo o mundo, possuindo incidência aumentada em crianças e idosos, consideradas como um grave problema na saúde pública, com cerca de 3,91 milhões de mortes no mundo em 2017 (LI X, et al., 2020). Forma esse conjunto de doenças, a doença pulmonar obstrutiva crônica (DPOC), asma e alergias respiratórias (BURNEY P, et al., 2015).

As DRCs manifestam um quadro clínico característico possuindo consideráveis efeitos na qualidade de vida dos indivíduos afetados (BURNEY P, et al., 2015). Há previsão de um aumento global de sua incidência, mesmo com os diagnósticos e tratamentos precoces nos países desenvolvidos (LI X, et al., 2020). No Brasil, em 2011, foi considerada a terceira maior causa de doenças crônicas não transmissíveis, correspondendo a mais de 6 milhões de internações no período de 2003 a 2013, sendo que a região sul obteve a maior taxa de internação do país com 410,6/100 mil habitantes em 2013 (BRASIL, 2016).

Constituindo o grupo das DRCs, a asma acomete cerca de 400 mil pessoas, compondo $2,5 \%$ do total de internações no Sistema Único de Saúde (SUS), além de ser responsável por 2.500 óbitos anualmente (BRASIL, 2016). No mundo, cerca de 300 milhões de pessoas sofrem de asma, sendo $60 \%$ crianças, por isso, esta doença é a mais comum na infância (DHARMAGE SC, et al., 2019).

A apresentação clínica é ampla e diversa, com uma história de sintomas respiratórios apresentando sibilos agudos e difusos, dispneia, tosse - sendo a manifestação mais frequente e persistente e, geralmente, mais intensa a noite com aperto no peito e desconforto torácico durante a crise (BUSH A, 2019).

A história familiar de doenças alérgicas e história de atopia são marcos importantes no desenvolvimento do quadro asmático (TOSKALA E e KENNEDY DW, 2015). A história social e fatores de riscos como, por exemplo, obesidade, tabagismo, hipersensibilidade a alérgenos, níveis séricos de lgE elevados, dermatite atópica, constituem-se como marcadores de gravidade para a exacerbação dos sintomas e podem ser investigados como fatores agravantes no quadro clínico e prognóstico (PROKOPAKIS E, et al., 2013).

Entende-se que a asma possui etiologia multifatorial, sendo desencadeada quando antígenos presentes no meio ambiente entram em contato com a mucosa das vias aéreas inferiores (VOSKAMP AL, et al., 2020). A exposição dos antígenos no sistema respiratório acaba por desenvolver uma resposta mediada por linfócitos T Helper $2\left(\mathrm{Th}_{2}\right)$ resultando na produção de anticorpos do tipo Imunoglobulina $\mathrm{E}$ (IgE) que se ligam aos mastócitos ativados e secretam citocinas como as interleucinas (IL-3, IL-4, IL-5, IL-10) e interferon-y (IFN-y), 
histamina, heparina, leucotrienos, eicosanoides, óxido nítrico e outros mediadores inflamatórios que resultam em hiperresponsividade brônquica (VOSKAMP AL, et al., 2020; MALTBY S, et al., 2017).

O processo inflamatório agride a musculatura lisa, causando edema da mucosa cursando com o aumento subsequente da produção e secreção de muco levando a um estreitamento brônquico paroxístico, que é reversível, resultando nas manifestações clínicas características da doença (MALTBY S, et al., 2017).

Geralmente o diagnóstico de asma é realizado antes dos 7 anos de idade em cerca de $75 \%$ dos casos, grande parte dos pacientes que receberam diagnóstico na infância terão remissão dos sintomas na puberdade (AALDEREN WM, 2012). Os pacientes que não estão em crise asmática, geralmente apresentam-se assintomáticos e com exame físico normal (CASTILLO JR, et al., 2017).

O principal exame complementar no diagnóstico da asma é a espirometria forçada, obtendo-se padrão de limitação ao fluxo aéreo e a reversibilidade por prova broncodilatadora (SAGLANI S e MENZIE-GOW AN, 2019). A interpretação do exame espirométrico para o diagnóstico depende de quais parâmetros são afetados, e atualmente o volume expiratório forçado no primeiro segundo $\left(V_{E F} F_{1}\right)$ devido a sua reprodutibilidade, tem sido a medida isolada mais eficaz para estabelecer a relação e gravidade de limitação ao fluxo aéreo e a resposta ao uso de broncodilatador (LAMBERT A, et al., 2015).

As diferentes apresentações clínicas do quadro podem ser compreendidas com o uso do exame espirométrico como, por exemplo, a obstrução ao fluxo aéreo observada em alguns asmáticos que é mensurada pelo índice de Tiffeneau ( $\mathrm{VEF}_{1} /$ capacidade vital forçada expiratória (CVF)) $<70 \%$ e a prova broncodilatadora é considerada positiva quanto se tem aumento em $200 \mathrm{~mL}$ e $12 \%$ na VEF 1 após o uso do broncodilatador. Espera-se que o VEF 1 esteja $<80 \%$, variações $>15 \%$ e $>400 \mathrm{~mL}$ no VEF 1 aumentam a especificidade, embora não haja consenso nesses valores (SINGH D, et al. 2019). A obstrução ao fluxo aéreo apresentado durante o quadro clínico é reversível espontaneamente ou com tratamento (FERREIRA DS, et al., 2018).

Outra patologia prevalente que compõe o grupo das DRCs é a bronquite, caracterizada por um processo inflamatório agudo ou crônico que acomete os brônquios, sem evidência de pneumonia (KINKADE S e LONG NA, 2016). O processo agudo pode afetar até $5 \%$ da população adulta, principalmente no inverno, responsável por mais de 10 milhões de consultas anuais (MARTÍNEZ MA, et al., 2014). Possui como principais agentes etiológicos os vírus, bactérias, alérgenos e a inalação de substâncias. Geralmente, os vírus são a causa mais comum, destacando os da Infuenza A e B (LIN SY e CHEN HW, 2017).

Alguns fatores de risco para desenvolvimento da bronquite aguda já estão consolidados como a história prévia de tabagismo, residir em local altamente poluído ou história pessoal de asma, sendo que em alguns casos específicos o quadro pode ser desencadeado por pólen, alérgenos e vapores (KARUNANAYAKE CP, et al., 2017). A avaliação clínica se baseia na história médica pregressa e no exame físico, especialmente do aparelho pulmonar (MARTÍNEZ MA, et al., 2014).

Já a bronquite crônica é definida como tosse com expectoração por pelo menos 3 meses ao ano, durante 2 anos consecutivos (KIM V, et al., 2016). Pode ter associação com o enfisema pulmonar, como parte do quadro de DPOC, tendo distúrbio ventilatório obstrutivo associado, com redução da CVF, não sendo responsivo ao broncodilatador (FERRÉ A, et al., 2012).

A saturação de oxigênio pode auxiliar no estadiamento da gravidade da doença, associada com a temperatura, frequências cardíaca e respiratória (MARTíNEZ MA, et al., 2014). Geralmente nenhuma investigação complementar é necessária se estes dados estiverem dentro da faixa da normalidade. 0 diagnóstico, da bronquite aguda ou crônica, é eminentemente clínico, consistindo na identificação de sinais de acometimento da árvore brônquica, especialmente sibilos, roncos e tosse (ALBERT RH, 2010).

O exame espirométrico não é item obrigatório para realizar diagnóstico de bronquite, entretanto quando realizado evidência hiperresponsividade brônquica transitória em $40 \%$ dos pacientes com quadro de bronquite aguda (NGU S, et al., 2019). O prognóstico destes pacientes é favorável, tendo remissão da obstrução ao fluxo do ar e da hiperresponsividade brônquica geralmente em 6 semanas, necessitando de acompanhamento 
ambulatorial, sendo raras às indicações de internações (NGU S, et al., 2019). É importante, neste cenário de pacientes, descartar pneumonia, principalmente nos pacientes que apresentam dispneia, expectoração purulenta, taquicardia, taquipneia, temperatura axilar $>37,8^{\circ} \mathrm{C}$, crepitações na ausculta pulmonar (BAI L, et al., 2018).

Diante da alta prevalência e incidência destas patologias, deve-se desenvolver estudos complementares para mapeamento epidemiológico e avaliação de prognóstico dos pacientes. Este estudo teve por objetivo avaliar os diferentes padrões espirométricos expressados na resposta ao broncodilatador em pacientes acometidos por doenças respiratórias como asma e bronquite descrevendo as características clínicasepidemiológicas avaliadas.

\section{MÉTODOS}

Foi realizado um estudo de campo de caráter analítico retrospectivo, caracterizando o perfil epidemiológico e espirométrico de pacientes com asma e bronquite que foram atendidos em consulta médica de rotina na especialidade de pneumologia em um Ambulatório Universitário no Vale do Taquari/RS, com aprovação, sem restrições, pelo Comitê de Ética em Pesquisa da Univates com o CAAE: 11435419.3.0000.5310.

Os testes espirométricos foram realizados utilizando um espirômetro (MiniSpir - Smart Medical). Durante a execução, realizou-se uma etapa pré-broncodilatador e uma etapa pós-broncodilatador, obtida 15 minutos após a administração de $400 \mathrm{mcg}$ de salbutamol por nebulímetro pressurizado. Os seguintes valores foram obtidos nos testes espirométricos e utilizados no estudo: 1) valor de $F V C, F E V_{1}, V_{E F} / F V C$ Prébroncodilatador; 2) valor de FVC, $\mathrm{FEV}_{1}, \mathrm{VEF}_{1} / \mathrm{FVC}$ Pré e Pós-broncodilatador. Os exames espirométricos preenchiam todos os critérios de aceitação e reprodução exigidos pela Sociedade Brasileira de Pneumologia e Tisiologia (SBPT).

Inicialmente, foram selecionados pacientes entre os anos de 2016-2019 que continham o diagnóstico clínico firmado de asma, bronquite ou rinite. O diagnóstico foi realizado pelo médico assistente da especialidade. No total, 56 pacientes foram incluídos. Após, foi analisado o prontuário e selecionado os pacientes que possuíam um exame de espirometria realizado no período indicado. Em seguida, 20 foram excluídos do estudo por não terem realizado um exame espirométrico e 2 por serem menores de 18 anos. Dos pacientes que fizeram o exame espirométrico, obteve-se a seguinte distribuição: 23 no grupo de asma, 8 no grupo de bronquite e 1 do grupo de rinite. No final, portanto averiguou-se que 34 pacientes correspondiam aos critérios estabelecidos.

Para análise dos dados, as variáveis quantitativas foram descritas por meio de medidas de tendência central e dispersão e as variáveis categóricas através de razões, proporções e taxas. As diferenças nas proporções foram avaliadas através do Teste t pareado e amostras independentes. As diferentes expressões de resposta ao broncodilatador foram correlacionadas com o valor basal do VEF 1 e a idade através da Correlação de Pearson ( $r$ ). Valores de $p$ foram considerados significativos se $\leq 0,05$.

Os valores espirométricos do paciente com rinite foram excluídos das análises estatísticas devido ao número amostral ser interpretado pelos autores deste estudo como muito reduzido, não possibilitando correlações clínico epidemiológicas satisfatórias. Para este trabalho não foi diferenciada os quadros de bronquite aguda e crônica do diagnóstico dos pacientes, pois o intuito foi comparar a patologia em geral com o quadro de asma. O software utilizado para análise dos dados foi o Statistical Package for the Social Sciences - SPSS versão 20.0 (SPSS Inc., Chicago, IL, EUA).

\section{RESULTADOS}

Dos 34 pacientes avaliados neste estudo, 23 (70\%) tinham diagnóstico de asma e $9(28 \%)$ de bronquite e $1(2 \%)$ de rinite. Em relação a idade e peso $15(45 \%)$ dos pacientes tinham entre 40-60 anos e em sua maioria do sexo feminino 19 (58\%) e possuíam peso considerado normal pelo índice de massa corporal (IMC). Além disso, 25 (45\%) não praticam atividade física e 29 (88\%) não fumavam (Tabela 1). 
Tabela 1 - Características gerais dos pacientes selecionados no período de 2016 - 2019 .

\begin{tabular}{cc}
\hline Variável & $\mathbf{N}(\%)$ \\
\hline Idade (média $\left.\pm \mathrm{ep}^{*}\right)$ & $46.96 \pm 3.66$ \\
\hline Sexo (F/M) & $19(58 \%) / 14(42 \%)$ \\
\hline Não fumantes & $29(88 \%)$ \\
\hline IMC (média \pm ep) & $26.24 \pm 1.3$ \\
\hline Sedentarismo & $25(45 \%)$ \\
\hline Sem resposta ao broncodilatador & $22(67 \%)$ \\
\hline Asma & $23(70 \%)$ \\
\hline Bronquite & $9(28 \%)$ \\
\hline Rinite & $1(2 \%)$ \\
\hline
\end{tabular}

Legenda: *erro padrão. Fonte: Vieira L, et al., 2021.

O quadro clínico dos pacientes foi semelhante. Os asmáticos se apresentaram com quadro clínico obstrutivo 11 (47,82\%), quanto a gravidade 8 (34,78\%) se apresentaram sem gravidade ou gravidade leve 8 (34,78\%). A maioria dos pacientes com bronquite se apresentou com quadro clínico obstrutivo 5 (55,55\%), sem gravidade 3 (33,33\%) ou gravidade moderada 3 (33,33\%). A maioria dos pacientes, no estudo, não teve resposta ao broncodilatador 22 (67\%) (Tabela 2$)$.

Tabela 2 - Características gerais do quadro clínico e gravidade dos pacientes selecionados no período de 2016-2019.

\begin{tabular}{|c|c|c|c|c|}
\hline Variável & Asma (n) & Bronquite (n) & $\mathbf{p}$ & $\mathbf{t}$ \\
\hline $\begin{array}{l}\text { Quadro clínico } \\
\text { Sem obstrução/Obstrutivo/Restritivo/Misto }\end{array}$ & $6 / 11 / 2 / 4$ & $2 / 5 / 0 / 2$ & 0.16 & 1.59 \\
\hline $\begin{array}{l}\text { Gravidade } \\
\text { Sem gravidade/Leve/Moderado/Grave }\end{array}$ & 8/8/4/3 & $3 / 2 / 3 / 1$ & 0.14 & 1.83 \\
\hline
\end{tabular}

Fonte: Vieira L, et al., 2021.

O valor médio de $V_{E F}$ (L) e $V F_{1} / C V F \%$ pré-broncodilatador em pacientes asmáticos não diferiu significativamente do valor de pós-broncodilatação dos mesmos parâmetros respectivamente $(p<0,12$; $\mathrm{p}<0,7856)$. Quando comparado os valores pré-FVC\% e pós-FVC\% no grupo asmático a diferença foi significativa $(p<0.0001)$ (Tabela 3).

Tabela 3 - Comparação das variáveis entre Pré e Pós resposta ao broncodilatador nos pacientes acometidos por Asma.

\begin{tabular}{|c|c|c|c|}
\hline Variável & Asma (média \pm ep) & $\mathbf{p}^{*}$ & $t$ \\
\hline Pré-FVC\% & $87.2 \pm 5.03$ & \multirow{2}{*}{$<0.0001$} & \multirow{2}{*}{5.69} \\
\hline Pós-FVC\% & $52 \pm 5.13$ & & \\
\hline Pré-VEF $1(L)$ & $74.8 \pm 4.34$ & \multirow{2}{*}{$<0.12$} & \multirow{2}{*}{-1.59} \\
\hline Pós-VEF ${ }_{1}(\mathrm{~L})$ & $77.3 \pm 4.46$ & & \\
\hline Pré-VEF $1 / C V F \%$ & $87.6 \pm 2.98$ & \multirow{2}{*}{$<0.78$} & \multirow{2}{*}{-0.27} \\
\hline Pós-VEF $1 / C V F \%$ & $87.9 \pm 3.22$ & & \\
\hline
\end{tabular}

Legenda: * Teste $t$ pareado. Fonte: Vieira L, et al., 2021.

Para a bronquite, não foram observadas diferenças nos padrões de expressão dos valores entre Pré e Pós o uso do broncodilatador ( $p=0,67 ; 0.93 ; 0.54$, respectivamente) (Tabela 4). 
Tabela 4 - Comparação das variáveis entre Pré e Pós resposta ao broncodilatador nos pacientes acometidos por Bronquite.

\begin{tabular}{|c|c|c|c|}
\hline Variável & Bronquite (média $\pm \mathrm{ep}$ ) & $\mathbf{p}^{\star}$ & $\mathbf{t}$ \\
\hline Pré-FVC\% & $88 \pm 8.23$ & \multirow[b]{2}{*}{0.67} & \multirow[b]{2}{*}{-0.43} \\
\hline Pós-FVC\% & $90.66 \pm 9.63$ & & \\
\hline Pré-VEF $1(L)$ & $68.22 \pm 7.5$ & \multirow{2}{*}{0.93} & \multirow{2}{*}{-0.08} \\
\hline Pós-VEF $1(L)$ & $68.66 \pm 8.61$ & & \\
\hline Pré-VEF $1 / C V F \%$ & $81.44 \pm 6.71$ & \multirow{2}{*}{0.54} & \multirow{2}{*}{0.65} \\
\hline Pós-VEF $1 /$ CVF\% & $79.11 \pm 7.53$ & & \\
\hline
\end{tabular}

Legenda: * Teste $t$ pareado. Fonte: Vieira L, et al., 2021.

Considerando os pacientes com asma e bronquite, a expressão pós-FVC demonstrou diferença significativa entre as duas patologias $(p<0,001)$. Houve maior variação nos valores de $V_{E F}$ nos pacientes asmáticos quando comparado com os portadores de bronquite (Tabela 5).

Tabela 5 - Comparação de cada variável Pré e Pós uso do broncodilatador entre os pacientes acometidos por Asma e Bronquite.

\begin{tabular}{|c|c|c|}
\hline Variável & $\mathbf{p}^{*}$ & $t$ \\
\hline Pré-FVC\% & 0.93 & -0.07 \\
\hline Pós-FVC\% & $<0.001$ & -3.8 \\
\hline Pré-VEF $F_{1}(\mathrm{~L})$ & 0.43 & 0.78 \\
\hline Pós-VEF $F_{1}(\mathrm{~L})$ & 0.34 & 0.96 \\
\hline Pré-VEF $1 / C V F \%$ & 0.33 & 0.97 \\
\hline Pós-VEF $1 / C V F \%$ & 0.21 & 1.27 \\
\hline
\end{tabular}

Legenda: * Teste $t$ amostras independentes. Fonte: Vieira L, et al., 2021.

\section{DISCUSSÃO}

Um forte diferencial neste trabalho é a construção de uma análise detalhada do perfil espirométrico de pacientes acometidos por doença respiratória em um ambulatório de especialidades médicas, visando o mapeamento profundo e elaboração de estratégias para aprimorar o seguimento clínico. Neste estudo, o principal diagnóstico clínico foi de asma, seguido de bronquite. Tal achado foi relatado em outras pesquisas, segundo o estudo multicêntrico Study for Asthma and Allergies in Childhood (ISAAC) realizado em 56 países, o Brasil ocupava a $8^{a}$ posição em prevalência de asma (BEASLEY R, 1998). Já o Departamento de Informática do Sistema Único de Saúde do Brasil (DATASUS), em 2011, colocou a asma como a quarta causa de internação no país (BRASIL, 2016).

De uma forma geral, no estudo não se obteve diferenças significativas na apresentação clínica entre os grupos de pacientes analisados. Analisando-se o Índice de Massa Corporal (IMC) a média observada (26.24 \pm 1.3$)$ configurou-se em sobrepeso. Algumas pesquisas estudaram a relação do IMC com a função pulmonar e os testes espirométricos preditivos que consideram em sua equação a idade e a altura para essa faixa de peso, parecem perder impacto uma vez que o IMC entre 20 e $30 \mathrm{~kg} / \mathrm{m}^{2}$ não causa grandes diferenças nos resultados obtidos nos testes (BHATTI U, et al., 2019). Tem-se bem relatado que em pacientes obesos o quadro de asma se apresenta com sintomas mais protuberantes e inflamação das vias aéreas (BAFFI CW, et al., 2015).

Um único estudo de coorte longitudinal prospectivo abordou a relação entre a obesidade e a hiperreatividade das vias aéreas em mais de 7.000 adultos, concluindo que o risco para desenvolver a hiperresponsividade não está diretamente ligado ao IMC, pois homens com baixo IMC também tiveram risco aumentado para desenvolvimento de hiperresponsividade (LITONJUA AA, et al., 2002).

Outros estudos, com um número menor de indivíduos, também não conseguiram determinar tal relação, muito provavelmente pelo tamanho da amostra, pelas diferenças ou composição do grupo de estudo 
(BUSTOS P, et al., 2005). Um estudo prévio já apontou diferenças significativas entre os eixos VEF 1 e FVC em pacientes obesos se comparado com eutróficos (BAFFI CW, et al., 2015). Em nosso estudo, o IMC médio de sobrepeso aparentemente não afetou os valores espirométricos obtidos, colaborando com os resultados das pesquisas prévias.

Já em relação aos valores espirométricos obtidos nos pacientes acometidos por asma não houve variação considerável no eixo pré e pós-VEF $\mathrm{V}_{1}$ em Teste T Pareado. Quando comparado com o grupo de pacientes com bronquite, houve uma maior variação de $\mathrm{VEF}_{1}$ no grupo dos pacientes asmáticos. Esse achado pode estar relacionado com o perfil clínico da asma, pois sabe-se que há uma associação da maioria das respostas isoladas de $\mathrm{VEF}_{1}$, cerca de $83 \%$, com os pacientes asmáticos e a sua variação isolada pós-broncodilatador é correlacionada com dilatação brônquica de aérea central (SILVESTRI IC, et al., 2008).

No eixo pós-FVC houve uma diferença considerável entre os pacientes com asma e bronquite sugerindo uma resposta e apresentação fisiopatológica distintas entre as duas doenças analisadas. Os pacientes com bronquite tiveram uma discreta mudança nos valores do FVC e VEF 1 quando comparado o pré e pósbroncodilatador, sem diferença estatística considerável e com variação menor quando comparado com a asma reforçando o nosso achado. É importante destacar que os valores permaneceram acima da relação para o diagnóstico de DPOC ( $\mathrm{VEF}_{1} / \mathrm{FVC}<0,7$ (pós-broncodilatador) (SINGH D, et al. 2019).

Nosso estudo demonstrou que a maioria dos pacientes asmáticos estudados apresenta quadro clínico com perfil obstrutivo. Dos pacientes analisados, 22 (67\%) não tiveram resposta ao uso de broncodilatador. O nosso achado corrobora com uma pesquisa prévia, na qual a maioria dos pacientes asmáticos apresenta baixa reversibilidade ao uso de broncodilatador, sendo que essa condição pode ocorrer principalmente no período inter crise ou nos pacientes clinicamente estáveis ou graves (AZEVEDO KRS, 2015).

Esse achado pode levantar uma consideração. É sabido que após o diagnóstico, a reversibilidade da obstrução pode ser avaliada medindo o VEF 1 pré e pós broncodilatador (GHOUL JE, et al., 2019), no entanto em nosso estudo não houve uma análise profunda do perfil clínico dos pacientes como, por exemplo, análise das evoluções médicas e dos tratamentos farmacológicos instituídos.

Quanto especificamente à resposta ao broncodilatador, JANSON et al. (2019) analisou VEF 1 e CVF de 35.628 indivíduos, sendo 2.833 asmáticos, 1.146 com doença pulmonar obstrutiva crônica (DPOC) e 31.649 sem patologia de vias aéreas. A reversibilidade, pós uso de broncodilatador, foi muito semelhante nos grupos de pacientes com asma e DPOC, indicando que o exame espirométrico possui limitações para distinguir entre estas duas patologias, no entanto quando a reversibilidade é expressa pode ser um forte marcador fenotípico de asma (JANSON C, et al., 2019). Sabe-se que o mecanismo de ação do broncodilatador se correlaciona inversamente com o grau de hiperressonância à adenosina 5'-monofostato (AMP) durante o desafio brônquico, que é conhecido por refletir a persistência da inflamação brônquica subjacente na asma (COX CA, et al., 2019).

Há evidências, ainda, de correlação entre a resposta ao broncodilatador e outros marcadores de inflamação das vias aéreas, como óxido nítrico e eosinófilos em amostras de biópsia brônquica ou uma combinação de IgE sérica, eosinófilos sanguíneos e óxido nítrico exalado (MAHESH PA, 2017). Tais evidências não foram estudadas em nosso estudo e, portanto, não conseguimos afirmar com exatidão o impactado desses componentes na resposta ao broncodilatador dos pacientes estudados.

De uma forma geral, o hábito de fumar independente se for ativo ou passivo, associa-se a uma diminuição da função pulmonar, com uma menor resposta ao tratamento com corticoides inalatórios e sistêmicos nos pacientes asmáticos (ZHENG X, et al., 2012). No estudo, 29 (88\%) dos pacientes não fumavam. Sabe-se que nos pacientes asmáticos não fumantes, a celularidade predominante são os eosinófilos; já nos pacientes asmáticos fumantes há um aumento do número de mastócitos e neutrófilos, identificados nas lavagens brônquicas, advindos muito provavelmente da fumaça do cigarro (BOTELHO FM, et al., 2011).

Em fumantes há uma modificação importante no metabolismo do ácido araquidônico nas vias da lipoxigenase quando comparado com os que nunca fumaram (THOMSON NC, et al., 2014). Essa característica confere ao grupo de fumantes uma pior resposta aos corticosteroides, além de aumento da 
morbidade e mortalidade, somado a sintomas mais graves, maior frequência de exacerbações e pior qualidade de vida (BELVISI MG, et al., 2018).

O fumante asmático faz parte muito provavelmente de um fenótipo especial com importantes implicações clínicas (BAKAKOS P, et al., 2016). Observamos em nosso estudo, uma normalidade na relação VEF $1 / F V C$. $O$ padrão encontrado na relação $\mathrm{VEF}_{1} / \mathrm{FVC}$ pode ser justificada pelo baixo número de pacientes fumantes, obtendo uma análise relativamente fraca nessa comparação. Somado a essa dificuldade, o IMC médio de sobrepeso, não permitiu uma observação e correlação clínica adequada acerca dos valores reduzidos de $\mathrm{VEF}_{1}$ e FVC.

\section{CONSIDERAÇÕES FINAIS}

Nosso estudo demonstrou uma alta incidência de asma nos pacientes atendidos em um ambulatório universitário, bem como a maioria dos exames espirométricos não possuem resposta ao broncodilatador e são de indivíduos com sobrepeso, ocorrendo maior variação de $\mathrm{VEF}_{1}$ no grupo dos pacientes asmáticos. Essas evidências são atribuídas ao fato de que a asma é uma doença heterogênea com diferentes fenótipos e das características da maioria dos indivíduos do grupo como, por exemplo, a prática de atividades físicas e o hábito de não fumar. Tais dados reforçam que a responsividade ao broncodilatador pode ter relação com a apresentação do quadro clínico, fenotípico da asma, estilo de vida e, principalmente, do índice de massa corporal. É notório que pelo baixo número de pacientes e por ser um centro de referência, os valores obtidos podem estar superestimados, todavia ainda se encontram de acordo com a literatura.

\section{AGRADECIMENTOS E FINANCIAMENTO}

Os autores agradecem à Universidade do Vale do Taquari (Univates) pelo fornecimento do material necessário para a realização deste estudo.

\section{REFERÊNCIAS}

1. AALDEREN WM. Childhood asthma: diagnosis and treatment. Scientifica (Cairo), 2012; 2012:674204.

2. ALBERT RH. Diagnosis and treatment of acute bronchitis. Am Fam Physician, 2010; 82:1345-1350.

3. AZEVEDO KRS. Teste de broncodilatação: a incorporação de novos parâmetros na sua avaliação. Pulmão RJ, 2015; 24(1):8-13.

4. BAFFI CW, et al. Asthma and obesity: mechanisms and clinical implications. Asthma Res Pract, 2015;1:1.

5. BAI L, et al. Exposure to traffic-related air pollution and acute bronchitis in children: season and age as modifiers. $J$ Epidemiol Community Health, 2018;72(5):426-433.

6. BAKAKOS P, et al. Smoking asthma phenotype: diagnostic and management challenges. Curr Opin Pulm Med, 2016;22(1):53-8.

7. BEASLEY R. Worldwide variation in prevalence of symptoms of asthma, allergic rhinoconjunctivitis, and atopic eczema: ISAAC. The International Study of Asthma and Allergies in Childhood (ISAAC) Steering Committee. Lancet,1998;351(9111):1225-1232.

8. BELVISI MG, et al. Modelling the asthma phenotype: impact of cigarette smoke exposure. Respir Res, 2018;19(1):89.

9. BHATTI U, et al. Effect of Body Mass Index on respiratory parameters: A cross-sectional analytical Study. Pak J Med Sci, 2019;35(6):1724-1729.

10. BOTELHO FM, et al. Cigarette smoke differentially affects eosinophilia and remodeling in a model of house dust mite asthma. Am J Respir Cell Mol Biol, 2011;45(4):753-60.

11. BRASIL. Perfil da morbimortalidade por doenças respiratórias crônicas no Brasil, 2003 a 2013. Ministério da Saúde, 2016;47(19).

12. BURNEY $P$, et al. The global burden of chronic respiratory disease in adults. The International Journal of Tuberculosis and Lung Disease, 2015;19(1): 10-20.

13. BUSH A. Pathophysiological Mechanisms of Asthma. Front Pediatr, 2019; 7:68.

14. BUSTOS $P$, et al. Is there a causal relation between obesity and asthma? Evidence from Chile. International journal of obesity, 2005;29(7):804-9.

15. CASTILLO JR, et al. Asthma Exacerbations: Pathogenesis, Prevention, and Treatment. J Allergy Clin Immunol Pract, 2017; 5(4):918-927.

16. COX $\mathrm{CA}$, et al. Factors associated with hyperresponsiveness to adenosine 5 '-monophosphate in healthy subjects. Allergy, 2019; 74(11):2268-2270. 
17. DHARMAGE SC, et al. Epidemiology of Asthma in Children and Adults. Front Pediatr,2019; 7:246.

18. FERRÉ A, et al. Chronic bronchitis in the general population: influence of age, gender and socio-economic conditions. Respir Med, 2012; 106(3):467-71.

19. FERREIRA DS, et al. Airway pathology in severe asthma is related to airflow obstruction but not symptom control. Allergy, 2018;73(3):635-643.

20. GHOUL JE, et al. Determining the optimal time to assess the reversibility of airway obstruction. Lung India, 2019; $36(2): 123-130$.

21. JANSON C, et al. Bronchodilator reversibility in asthma and COPD: findings from three large population studies. Eur Respir J, 2019;54(3):1900561.

22. KARUNANAYAKE CP, et al. Bronchitis and Its Associated Risk Factors in First Nations Children. Children (Basel), 2017;4(12):103

23. KIM V, et al. Persistent and Newly Developed Chronic Bronchitis Are Associated with Worse Outcomes in Chronic Obstructive Pulmonary Disease. Ann Am Thorac Soc, 2016; 13(7):1016-25.

24. KINKADE S, LONG NA. Acute Bronchitis. Am Fam Physician, 2016; 94:560-565.

25. LAMBERT A, et al. Diagnostic accuracy of FEV1/forced vital capacity ratio z scores in asthmatic patients. J Allergy Clin Immunol, 2015;136(3):649-653.

26. LI X, et al. Trends and risk factors of mortality and disability adjusted life years for chronic respiratory diseases from 1990 to 2017: systematic analysis for the Global Burden of Disease Study 2017. BMJ, 2020; 368:m234.

27. LIN SY, CHEN HW. Infectious Bronchitis Virus Variants: Molecular Analysis and Pathogenicity Investigation. Int J Mol Sci, 2017; 18(10):2030.

28. LITONJUA AA, et al. Association of body mass index with the development of methacholine airway hyperresponsiveness in men: The Normative Aging Study. Thorax, 2002;57(7):581-5.

29. MAHESH PA. Evaluation of asthma severity: Relevance of total serum IgE, sputum and peripheral eosinophilia. Lung India, 2017;34(3):290-291.

30. MALTBY S, et al. Mouse models of severe asthma: understanding the mechanisms of steroid resistance, tissue remodelling and disease exacerbation. Respirology, 2017; 22(5):874-885.

31. MARTÍNEZ MA, et al. Protocolo diagnóstico y tratamiento empírico de la bronquitis aguda. Medicine (Madr), 2014;11(66):3963-3965.

32. NGU S, et al. Rhinovirus-induced Rapidly Progressing Acute Respiratory Distress Syndrome in an Immunocompetent Host. Cureus, 2019;11(2):e3997.

33. PROKOPAKIS E, et al. The pathophysiology of the hygiene hypothesis. Int J Pediatr Otolaryngol, 2013; 77:10651077.

34. SAGLANI S, MENZIE-GOW AN. Approaches to Asthma Diagnosis in Children and Adults. Front Pediatr, $2019 ; 7: 148$.

35. SINGH D, et al. Global Strategy for the Diagnosis, Management, and Prevention of Chronic Obstructive Lung Disease: the GOLD science committee report 2019. Eur Respir J, 2019; 18;53(5):1900164.

36. SILVESTRI IC, et al. Comparação da variação de resposta ao broncodilatador através da espirometria em portadores de asma ou doença pulmonar obstrutiva crônica. J. bras. pneumol, 2008; 34(9):675-682.

37. THOMSON NC, et al. Arachidonic acid metabolites and enzyme transcripts in asthma are altered by cigarette smoking. Allergy, 2014; 69(4):527-36.

38. TOSKALA E, KENNEDY DW. Asthma risk factors. Int Forum Allergy Rhinol, 2015; 1(1):S11-6.

39. VOSKAMP AL, et al. Modulating local airway immune responses to treat allergic asthma: lessons from experimental models and human studies. Semin Immunopathol, 2020; 42(1):95-110.

40. ZHENG X. Smoking influences response to inhaled corticosteroids in patients with asthma: a meta-analysis. Curr Med Res Opin, 2012; 28(11):1791-8. 